\title{
Assessment of Flow Mediated Dilation by Pulse Wave Conduction Delay
}

\author{
Junichiro Hayano*, Yutaka Yoshida, Emi Yuda \\ Nagoya City University Graduate School of Medical Sciences, Nagoya, Japan. \\ * Corresponding author. Tel.: +81-52-853-8507; email: Hayano@med.nagoya-cu.ac.jp \\ Manuscript submitted August 27, 2017; accepted January 14, 2018. \\ doi: 10.17706/ijbbb.2018.8.3.147-154
}

\begin{abstract}
This study aimed at developing a new method for assessing flow mediated dilation (FMD) by bilateral differences in pulse conduction time. Delay in pulse conduction time (PCD) that accompanied FMD induced by forearm reactive hyperemia with 5-min avascularization was evaluated. PCD increased with FMD but the correlation coefficient between PCD and FMD was 0.33. PCD may have been influenced by unmeasured factors other than FMD.
\end{abstract}

Key words: Endothelial function, flow mediated dilation, pulse wave velocity, reactive hyperemia.

\section{Introduction}

Flow-mediated dilatation (FMD) is a fundamental endothelial function that regulates vessel diameter by releasing nitric oxide (NO) in response to increased sheer stress caused by increased blood flow [1]-[3]. Impairment of FMD is an earlier manifestation of atherosclerosis [4] and a predictor of adverse cardiovascular events [5], [6]. Currently, the most popular technique to study FMD is a non-invasive ultrasound-based method to detect dilation of the brachial artery induced by reactive hyperemia in peripheral vessels. This method, however, requires high performance ultrasonic equipment and sophisticated technologies [7] and even minor changes in the methodological approach may critically impact variability and decrease reproducibility of the FMD [8]. Considering the usefulness of FMD, development of more robust and non-skill dependent method is desired.

In the present study, a novel method was developed for estimating FMD from pulse wave conduction delay caused by FMD of the brachial artery. Arterial dilation by FMD is thought to be associated with an increase in radius and a decrease in wall thickness of arteries, both of which cause theoretically a reduction in pulse wave velocity (PWV). Furthermore, to detect a slight change in the pulse wave conduction time (PCT), PCT was measured simultaneously at the opposite arm as a control and was subtracted from the PCT in the arm in which FMD was induced. This method was thought to be useful for excluding the influences of systemic factors on peripheral arterial systems and emphasizing the effect of local factor, i.e., FMD. In this study, the relative pulse wave conduction delay (PCD) caused by reactive hyperemia by this method was compared with FMD simultaneously measured by ultrasound method in the same arm.

\section{Principle}

\subsection{Estimation of FMD by PCT}

FMD of peripheral arteries may be assessed by the changes in beat-to-beat PCT. According to the 
Moens-Korteweg formula, PWV is the function of vascular diameter, wall thickness, and its elasticity (Fig. 1). Release of NO, the mechanism of FMD, causes relaxation of vascular smooth muscle, which decreases elasticity of the vascular wall. The subsequent vasodilation would be accompanied by not only increase in arterial diameter but also decrease in wall thickness. All of these changes are expected to reduce PWV and to increase PCT.

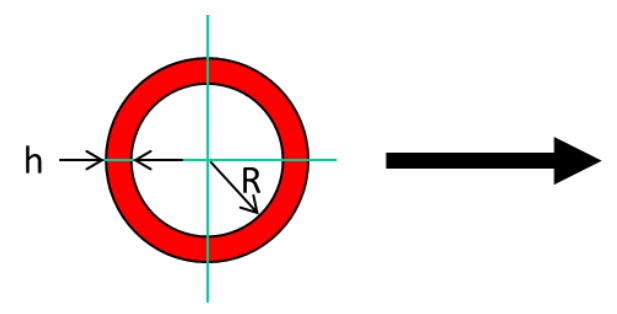

Control

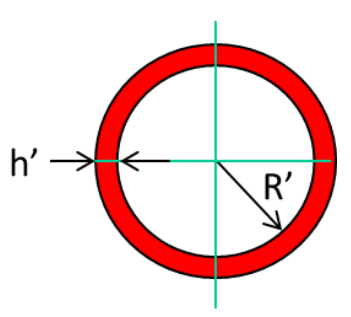

FMD

Fig. 1. Schema of the changes in cross-sectional view of an artery with flow mediated dilation (FMD).

In this model, the PWV for control is written as:

$$
P W V=\sqrt{\frac{E h}{2 \rho R}}
$$

and that for FMD is written as:

$$
P W V^{\prime}=\sqrt{\frac{E^{\prime} h^{\prime}}{2 \rho R^{\prime}}}
$$

where $E$ and $E^{\prime}=$ Young's modulus, $h$ and $h^{\prime}=$ arterial wall thickness, and $R$ and $R^{\prime}=$ arterial internal radius for control and FMD, respectively, and $\rho=$ blood density. When pulse wave conduction distance is assumed as D, PCT for control is

$$
P C T=\mathrm{D} \sqrt{\frac{2 \rho R}{E h}}=D \sqrt{\frac{4 \pi \rho}{A E}} R
$$

and that for FMD is

$$
P C T^{\prime}=\mathrm{D} \sqrt{\frac{2 \rho R^{\prime}}{E^{\prime} h^{\prime}}}=D \sqrt{\frac{4 \pi \rho}{A E^{\prime}}} R^{\prime}
$$

where $A=$ cross-sectional area of arterial wall, which is thought to be unchanged with FMD, and $E^{\prime} \approx E$ when arterial radius reached equilibrium with blood pressure. Then difference in PCT with FMD $(\triangle \mathrm{PCT})$ is

$$
\triangle P C T=P C T^{\prime}-P C T \approx D \sqrt{\frac{4 \pi \rho}{A E}}\left(R^{\prime}-R\right)=D \sqrt{\frac{4 \pi \rho}{A E}} \Delta R
$$

Thus, PCD is 


$$
P C D(\%)=100 \times \frac{\Delta P C T}{P C T}=100 \times \frac{\Delta R}{R}(\%)=F M D(\%)
$$

indicating that FMD can be estimated by PCD.

Because FMD would occur at all portion of arteries from the heart to the region of reactive hyperemia, the reduction of PWV can be expected for the entire length of these arteries. When arteries' diameters increase by an average of $0.5 \mathrm{~mm}$, the expected $\triangle \mathrm{PCT}$ from the heart to radial artery is estimated as $\approx 9 \mathrm{~ms}$ by this model.

\subsection{Cancellation of the Effects of Systemic Factors}

The arterial diameter is not only pulsating but also fluctuating spontaneously and changing in response to internal and external stimuli. The arterial diameter is dependent on heart rate and blood pressure, both of which show short-term variations such as those relating respiration and Mayer wave (10-second rhythm). Also, neuro-humoral, thermoregulatory and emotional factors (which could accompany arterial occlusion procedure to induce reactive hyperemia) affect the vascular tone directly and indirectly through heart rate, blood pressure, and respiration. The influences of these systemic factors confound the assessment of endothelium-dependent vascular responses and could contribute to the variation in FMD.

The reliability for measuring the change in PCT caused by FMD may be increased by contrasting the measures against those of the symmetric region of the arteries (the left arm; Fig. 2). For this purpose, time from ECG R-wave to pulse wave arrival to the radial arteries (PAT), which reflects theoretically isovolemic contraction time (ICT) + PCT, is measured simultaneously in both arms. Reactive hyperemia is induced in the right arm by cuff inflation and deflation at the forearm. The time-dependent changes in PCT $(\triangle P C T(t))$ specifically with FMD in the right arm can be measured as the difference in $\operatorname{PAT}(\mathrm{t})$ between right and left arms

$$
\Delta P C T(t)=P A T_{\text {right }}(t)-P A T_{\text {left }}(t)
$$

because ICT is common to both sides.

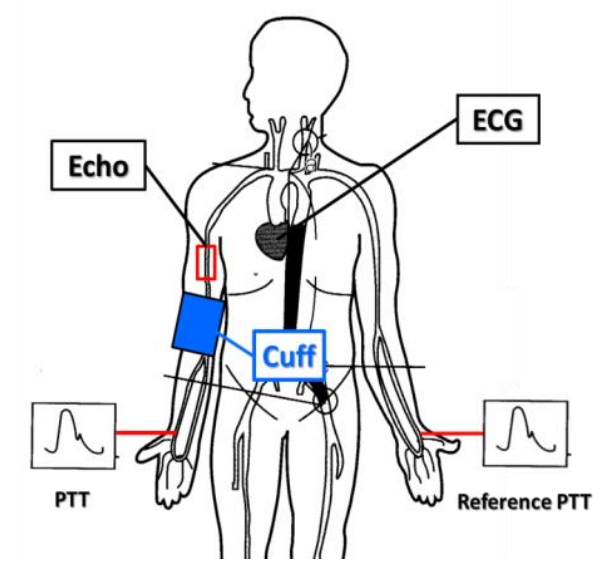

Fig. 2. Schema of measurement system.

\section{Method}

\subsection{Subjects}

The study subjects were 19 healthy peoples (13 males and 5 females; age 17-35 years). All of them gave 
written informed consent to participate in this study. The study was performed according to the protocol that has been approved by the Ethics Review Committee of Nagoya City University Graduate School of Medical Sciences (No. 742).

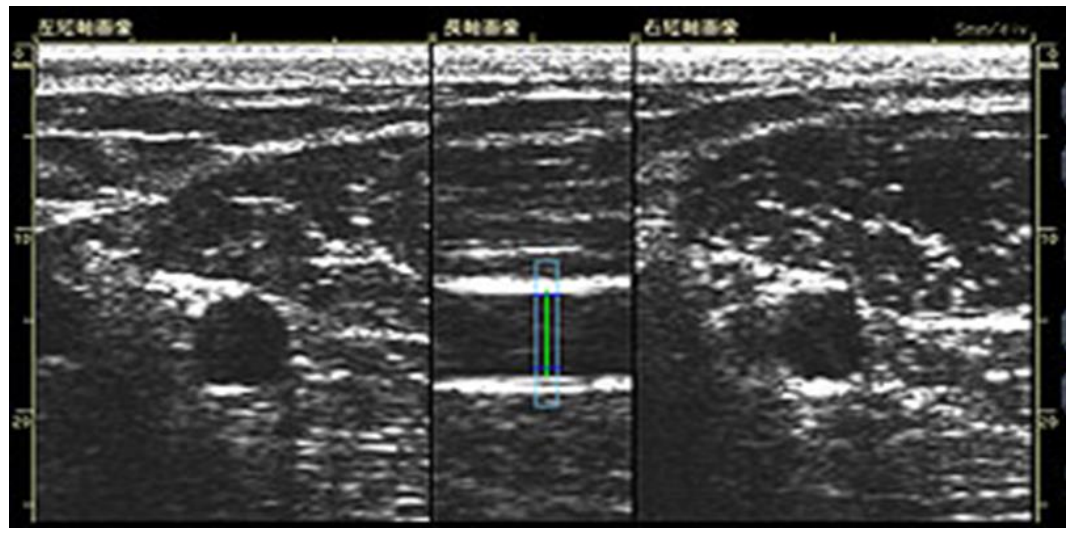

Fig. 3. Representative ultrasound image of the brachial artery. Both side panels show cross-sectional and center panel shows longitudinal views. Light blue line in the center panel is arterial diameter detected automatically by the system.

\subsection{Measurements}

Fig. 2 shows the schema of measurements. ECG electrodes for II lead were attached and infrared transdermal photoelectric pulse wave sensors (P-endo21, CCI Co., Ltd., Tokyo, Japan) were placed on the right and left radial arteries at the wrists. Pulse wave and ECG signals were digitalized at $1 \mathrm{kHz}$ and continuously stored in a personal computer. FMD of the brachial artery were assessed with semiautomatic ultrasound system (UNEXEF18G, Unex Co. Ltd., Nagoya, Japan). A cuff of sphygmomanometer for avascularization was placed around the right forearm and a $10-\mathrm{MHz}$ linear array transducer probe was fixed with a dedicated fixing device so that the longitudinal central tomographic image of the right brachial artery was recorded (Fig. 3). Using image-processing software equipped with the devise, diastolic arterial diameter was measured during experiment.

\subsection{Protocol}

Assessment of FMD was performed according to the guideline. [7] Subjects were instructed to fast for at least 12 hours before testing and to abstain from smoking and ingesting alcohol, caffeine or antioxidant vitamins prior to experiment. They were placed on a bed in the supine position. After attaching the equipment for measurements, 5-minute baseline data were recorded. Then, the right forearm cuff was inflated to a pressure $50 \mathrm{~mm} \mathrm{Hg}$ above the systolic blood pressure of each subject. The cuff pressure was maintained for 5 minute and deflated suddenly. Recording was continued for 6 more minutes after the cuff deflation for the period during which reactive hyperemia is assumed to occur.

\subsection{Data Analysis}

Using pulse wave signals from both sides of radial arteries, beat-to-beat PAT was measured as the time from ECG R wave to the foot point of each pulse wave. $\triangle \mathrm{PCT}$ was calculated as the difference between left and right PAT. Also, PCD (\%) was calculated as $100 \times\left(\triangle P C T_{\text {max }}-\triangle P C T_{\text {base }}\right) / \overline{P C T}_{\text {base }}$ where $\triangle P C T_{\text {base }}$ is $\triangle \mathrm{PCT}$ before cuff inflation, $\triangle P C T_{\max }$ is the maximum value of $\triangle \mathrm{PCT}$ during reactive hyperemia, and $\overline{P C T}_{\text {base }}$ is the average of left and right PAT before cuff inflation minus ICT, where a constant value of $33 \mathrm{~ms}$ was used for ICT in this study. 
From the diastolic diameter of the right brachial artery semi-automatically determined by the image-processing software, FMD was estimated as the percent change in the diameter over the baseline value at maximal dilation during reactive hyperemia.

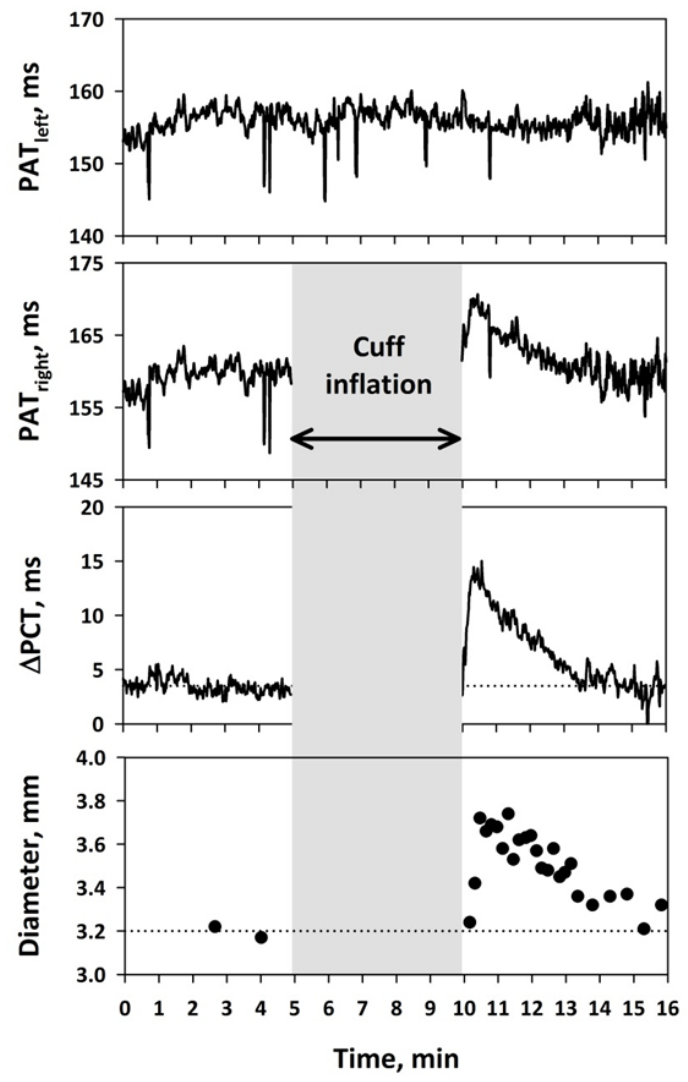

Fig. 4. Pulse arrival times (PAT) of left and right radial arteries and the changes in pulse conduction time $(\triangle \mathrm{PCT})$ as the difference between PAT of both sides in a representative subject (44-yr female). The lower most panel shows brachial artery diameter measured from ultrasound image.

\subsection{Statistical Analysis}

The relationship between PCD (\%) and FMD (\%) was evaluated with Pearson's product moment correlation coefficient. The agreement of these values were estimated with Bland and Altman plot [9].

\section{Results}

PAT on the right side (cuff side) showed a transient increase after release of cuff pressure with the peak increase occurring about 1 min after the release (Fig. 4). Although the PAT on the left side (control) showed no specific changes with cuff inflation and deflation, there observed considerable fluctuations at various frequencies (from slow trends to sharp spikes). Such fluctuations in PAT occurred synchronously between right and left sides, indicating they were caused by systemic factors, and they were subtracted out in the difference between them $(\triangle \mathrm{PCT})$. The cancellation procedure of systemic effects seemed quite effective when the PAT shows large spontaneous fluctuations (Fig. 5), where the changes in PAT after the cuff release were unclear in the raw PAT signal, but they were clearly observed in $\triangle$ PCT.

Both PCD and FMD were measured in 17 out of 19 subjects. In 2 subjects, PCD was not obtained due to technical error. As shown in Fig. 6, however, PCD showed positive but modest correlation with FMD ( $r=$ $0.58, P=0.01$ ). Also, Bland and Altman plot showed that the limits of agreement between PCD and FMD 
were from -5.7 to $5.5 \%$ with a systemic difference of $-0.1 \%$ (PCD < FMD). No significant effect was found for age, sex, or FMD on the difference between PCD an FMD.

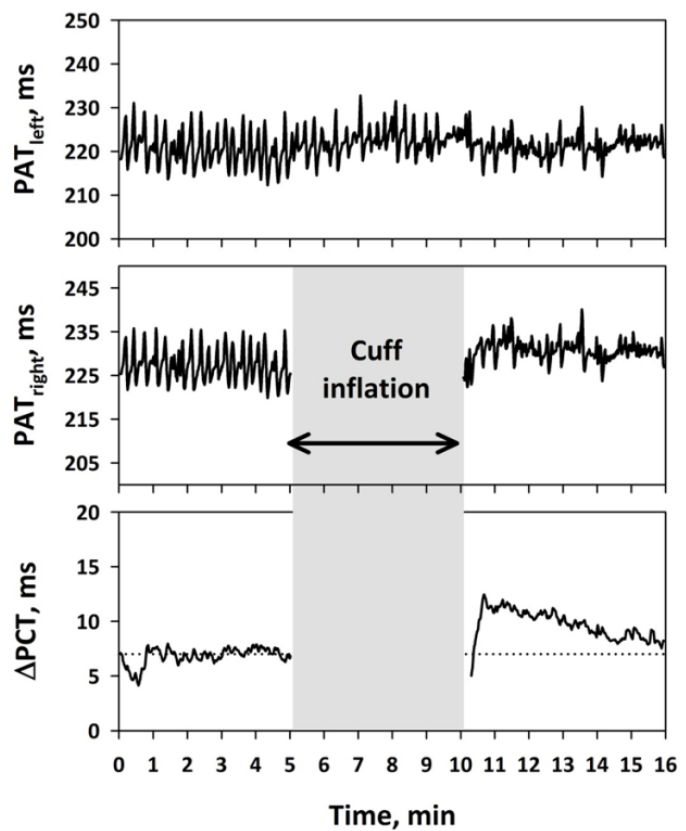

Fig. 5. Cancellation of systemic effects by the subtraction of PAT at the opposite-arm radial artery in a representative subject (32-yr male). The changes in PAT after the cuff release were unclear in the raw PAT signal, but they were clearly observed in $\triangle \mathrm{PCT}$.
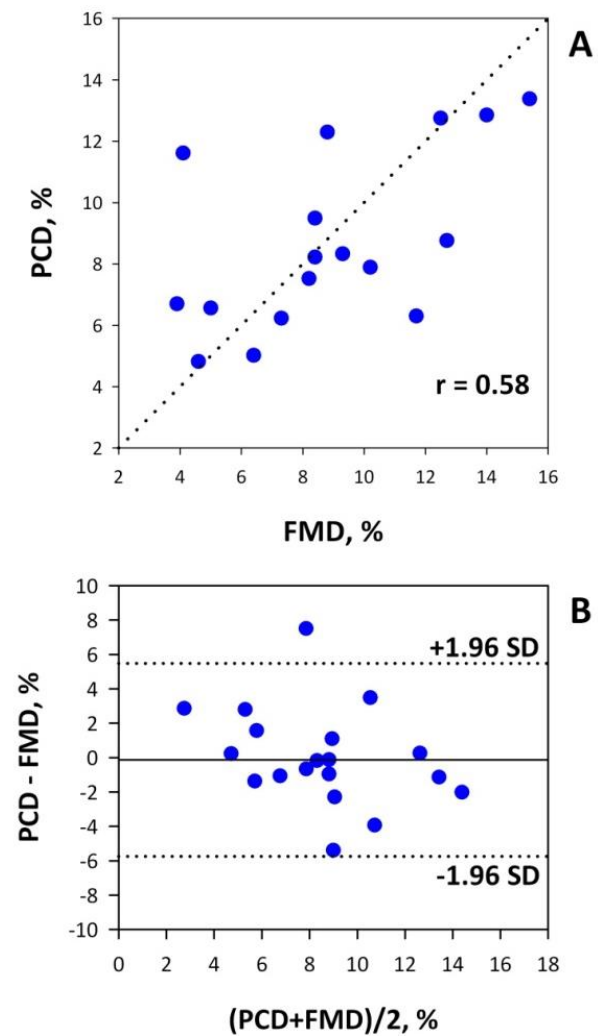

Fig. 6. Relationship between PCD and FMD measure by ultrasound image (A) and Bland and Altman plot for degree of agreement between them. 


\section{Discussions}

In this study, a new method was reported for assessing FMD by bilateral differences in PCT. The method measures the delay in PAT that accompanied FMD induced by forearm reactive hyperemia with 5-min avascularization. The usefulness of our method was evaluated by comparing with FMD assessed by the method of ultrasound vascular imaging. PCD increased with FMD but the correlation coefficient between PCD and FMD was 0.33. PCD may have been influenced by unmeasured factors other than FMD.

Our method seems advantageous over other methods including ultrasound vascular imaging in at least three points. First, $\triangle$ PCT would provide a sensitive and specific assessment of the local FMD response, because the influences of the systemic changes in hemodynamic and neurohumoral factors common to both sides are subtracted out. Second, the measurement of PCT requires no skillful technique. Third, the apparatus necessary for PCT is inexpensive.

The results of this study demonstrate the feasibility of the assessment of FMD by PCT. The PCT from ECG R-wave to the radial artery showed a transient increase during reactive hyperemia induced by 5 -min cuff inflation and following deflation. The time course of the response of PCT seems consistent with FMD and was actually similar to the change in the diameter of the brachial artery assessed by ultrasound echo imaging. Furthermore, using the PAT on the opposite side as control, the systemic fluctuations in PCT were effectively subtracted out and the changes in PCT specific to FMD can be clearly extracted. Because measurement of pulse wave requires neither specific skill nor expensive equipment, this technology seems to have potential as an assessment of FMD in practice.

The present study also determined the problems that need to be resolved. We found that PCD increased with FMD but the correlation coefficient between PCD and FMD was 0.33. PCD may have been influenced by unmeasured factors other than FMD. In the principle of this method, it was necessary to assume that Young's modulus E does not change with vasodilation. This assumption, however, may not appropriate because vasodilation occurs with relaxation of arterial wall smooth muscle, which may reduce the elasticity of vascular wall. Also, we used a constant value of ICT (33 ms) to estimate $\overline{P C T}_{\text {base }}$ that is the denominator for the calculation of \%PCD. ICT, however, may have individual difference that cannot be ignored. These factors may explain at least partly the low correlation coefficient between \%PCT and \%FMD.

\section{Acknowledgment}

This work was supported by the grant of the Knowledge Hub of Aichi, Japan [the Priority Research Project, P3-G1-S1-2b (J. Hayano)] and the Japan Society for the Promotion of Science, Japan [Grant-in-Aid for Scientific Research (C) 15590765 (J. Hayano)].

\section{References}

[1] Furchgott, R. F., \& Zawadzki, J. V. (1980). The obligatory role of endothelial cells in the relaxation of arterial smooth muscle by acetylcholine. Nature, 288, 373-376.

[2] Ignarro, L. J., Buga, G. M., Wood, K. S., Byrns, R. E., \& Chaudhuri, G. (1987). Endothelium-derived relaxing factor produced and released from artery and vein is nitric oxide. Proceedings of the National Academy of Sciences of the United States of America 84 (pp. 9265-9269).

[3] Behrendt, D., \& Ganz, P. (2002). Endothelial function. From vascular biology to clinical applications. American Journal of Cardiology, 90, 40L-48L.

[4] Deanfield, J. E., Halcox, J. P., \& Rabelink, T. J. (2007). Endothelial function and dysfunction: testing and clinical relevance. Circulation, 115, 1285-1295.

[5] Ras, R. T., Streppel, M. T., Draijer, R., \& Zock, P. L. (2013). Flow-mediated dilation and cardiovascular risk prediction: a systematic review with meta-analysis. International Journal of Cardiology, 168, 344-351. 
[6] Inaba, Y., Chen, J. A., \& Bergmann, S. R. (2010). Prediction of future cardiovascular outcomes by flow-mediated vasodilatation of brachial artery: a meta-analysis. Int J Cardiovasc Imaging, 26, 631-640.

[7] Corretti, M. C., Anderson, T. J., Benjamin, E. J., Celermajer, D., Charbonneau, F., Creager, M. A., Deanfield, J., Drexler, H., Gerhard-Herman, M., Herrington, D., Vallance, P., Vita, J., \& Vogel, R. (2002). Guidelines for the ultrasound assessment of endothelial-dependent flow-mediated vasodilation of the brachial artery: A report of the International Brachial Artery Reactivity Task Force. Journal of the American College of Cardiology, 39, 257-265.

[8] Greyling, A., Mil, A. C., Zock, P. L., Green, D. J., Ghiadoni, L., Thijssen, D. H., Dilation, T. I. W. G. O. F. M. (2016). Adherence to guidelines strongly improves reproducibility of brachial artery flow-mediated dilation. Atherosclerosis, 248, 196-202.

[9] Bland, J. M., \& Altman, D. G. (1986). Statistical methods for assessing agreement between two methods of clinical measurement. Lancet, 1, 307-310.

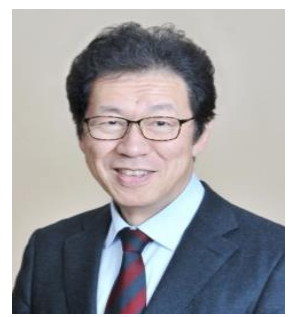

Junichiro Hayano graduated Nagoya City University Medical School, Nagoya, Japan and received the M.D. degree in 1980. From 1981 to 1983, he received residency trainings of psychosomatic medicine in Kyushu University School of Medicine, Fukuoka, Japan. He obtained the Ph.D. degree (Dr. of Medical Science) in 1988 from Nagoya City University Graduate School of Medical Sciences. From 1990 to 1991, he was working as a visiting associate at the Behavioral Medicine Research Center, Duke University Medical Center, Durham, NC, USA. In 1984, he got a faculty position at Nagoya City University Medical School and has been a Professor of Medicine at Nagoya City University Graduate School of Medical Sciences since 2003. His current interests are applications of dynamic electrocardiography and bio-signal monitoring to cardiology and health sciences.

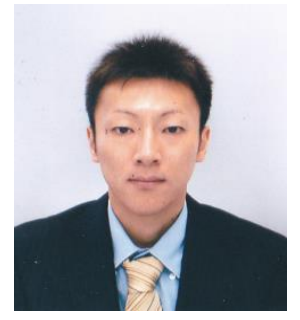

Yutaka Yoshida was born in Aichi, Japan in 1977. He received the B.S. and M.S. degrees in engineering from the Daido University, and Ph.D. degree in information management from Aichi Institute of Technology University, Nagoya, Japan, in 2008. From 2008 to 2012, he was a Research assistant with the Nagoya City University Graduate School of Arts and Sciences. From 2012 to 2016, he was a Project Researcher of "Center of Knowledge", with the Nagoya City University Graduate School of Medical sciences. Since 2016, he has been a Research engineer with the Nagoya City University Graduate School of Medical sciences. His research interests include Bioinformatics engineering and Digital signal processing.

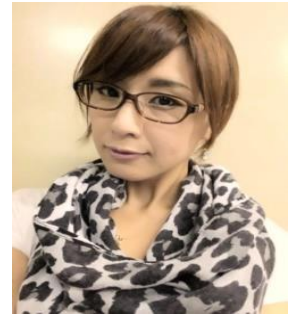

Emi Yuda was born in Mitaka City, Tokyo Japan in 1980. She studied computer science at Moscow University until 2003 and received M.S. degree from Tsukuba University, Japan. She will receive Ph.D. degree from Nihon University graduate school. From 2013 to 2014 she was a research assistant at the Santa Monica College Computer Science department in California, USA. Since 2015, she has been a Project Researcher of New Energy and Industrial Technology Development Organization (NEDO), with the Nagoya City University Graduate School of Medical sciences. Her research is Informatics and Intellectual property. Since 2017 she has been a member of the Steering Committee of the Electronic Intellectual Property Study Group of Information Processing Society of Japan. 